\title{
COMMENTARY
}

\section{The Role of Educators in Supporting the Mental Wellbeing of Postgraduate Pharmacist Distance Learners}

\author{
Zeenat Hassam, MPharm, ${ }^{\mathrm{a}}$ Nargis Gulzar, MPharm, ${ }^{\mathrm{a}}$ Asam Latif, $\mathrm{PhD}^{\mathrm{b}}$ \\ ${ }^{\text {a }}$ De Montfort University, School of Pharmacy, Faculty of Health and Life Sciences, Leicester, England \\ ${ }^{\mathrm{b}}$ University of Nottingham, School of Health Sciences, Faculty of Medicine and Health Sciences, Nottingham, \\ England
}

Corresponding Author: Zeenat Hassam, De Montfort University, School of Pharmacy, Faculty of Health and Life Sciences, Hawthorn Building, The Newark, LE2 7BY, Leicester, England. Tel: 01162577129. Email: zeenat.h.hassam@dmu.ac.uk

Submitted August 13, 2021; accepted November 29, 2021; ePublished December 2021

Front-line healthcare professionals have experienced rapid changes to workload and pressures during the COVID-19 pandemic, resulting in anxiety, depression and mental health stressors. For working professionals engaged in pharmacy postgraduate distance learning, access to educators was seen as a means to relay some of these stories and offload the stress caused by these unprecedented circumstances. The postgraduate pharmacy education team at De Montfort University felt a moral responsibility to provide extra support and extended their roles towards offering greater wellbeing support. In this commentary we detail the emergence of this new role and offer insights into how this was fashioned and its significance for catering to the mental health needs of pharmacists. This role has largely gone undetected and research is needed into the acceptability and feasibility of such a model and its plausibility and sustainability in the long term.

Keywords: clinical pharmacy, mental health, wellbeing, covid-19, student support

\section{INTRODUCTION}

The COVID-19 pandemic radically impacted educational institutions at all levels resulting in closures, suspension of face-to-face teaching and urgent reconfiguration of learning. ${ }^{1}$ For educators, the pandemic accelerated pedagogical innovations and the use of digital learning tools. Didactic teaching practices have been set aside for a more blended approach emphasising connectivity, flexibility and interactivity of learners. ${ }^{2-4}$ Whilst such teaching methodologies have been well reported in the literature, one important yet under-investigated facet of learning is the impact on professional roles of educators themselves. Specifically, how during the pandemic, the role of the educator has been influenced by healthcare professional's engagement with distance learning and the tacit changes that have emerged in the student-educator relationship. Our particular interest is how educators have acknowledged the pressures the pandemic has put on working pharmacists and subsequently responded by taking on greater wellbeing responsibilities to support learners' mental health. In this article, we unpack this phenomenon by illustrating how educators involved in the delivery of a postgraduate Clinical Pharmacy programme at one Higher Education Institution (De Montfort University) responded by taking on this unforeseen role. We discuss how these interactions implicitly formed in the immediate aftermath of lockdown and how this led to educators adopting a more purposeful wellbeing supportive role. We postulate whether these new roles should be sustained, developed or further promoted as a means to offset the increasing workload pressures and demands of postgraduate professionals.

\section{DISCUSSION}

Unquestionably, the pandemic has had a significant impact on the mental health of healthcare professionals. The strain of workload pressures, concerns about transmission and challenging care decisions have contributed towards increased reports of poor mental health. An umbrella review of meta-analyses outlined that the prevalence of anxiety and depression experienced during the pandemic has been reported at $24.9 \%$ and $24.8 \%$, respectively. ${ }^{5}$ From the studies, all of which were published in 2020, some referred to 'healthcare workers' in general and others outlined specific professions including nurses, physicians, social workers etc. Reports also suggest health professionals may have low interest in seeking specialist help, preferring to take responsibility for their own mental health. ${ }^{6}$ In pharmacy, pharmacists and support staff have experienced rapid changes to workload and workflow, with some feeling unprepared and exhausted resulting in anxiety, insomnia, post-traumatic stress disorder and/or depression. ${ }^{7}$ Despite this they continue to prioritise the needs of others sometimes to the detriment of their own wellbeing. ${ }^{8}$ The repeated exposure to psychological distress places the pharmacy workforce at risk of long-term psychological trauma and burnout. ${ }^{9}$ There have been calls to guarantee easier access to support structures to lessen the psychological burden on frontline healthcare workers. ${ }^{10}$ However, for those pharmacists undertaking postgraduate education alongside their 
clinical roles, be it through motivation driven by a desire for professional development, improving patient outcomes or contractual requirements, Covid-19 adds an extra layer of complexity. ${ }^{11}$ These difficulties are further compounded by reported isolation and loneliness that students are at risk of feeling. ${ }^{12}$ All of these factors collectively impact on a students' ability to perform successfully during their studies. ${ }^{13}$

The existing supportive relationship between educators and students is described through a range of terminologies: academic tutor, personal tutor, mentor etc. This relationship is formed when students are designated a named educator who acts as the first port of call for students to seek support and guidance. For the majority of institutions, the role can be diverse, comprising of a blend of academic support or guidance, career planning and pastoral support. It is unsurprising that there has been little theorising of what this supportive role actually is. The interactionalist theory is one of the more notable positions regarding this relationship through the need for students to have both formal and informal integration within academic systems to enhance retention. Atkinson, ${ }^{14}$ calls for this role to be redeveloped or reviewed in light of the changing context of provision. The landscape across higher education has been gradually shifting online and students can no longer be assumed to be resident. It may be argued that distance learning education requires a new model of student support altogether. Changes made to distance learning programmes in light of the pandemic resulted in the few face-to-face contact days also being delivered remotely. Some programmes sought to support student resilience through building relationships and networks between students and educators. ${ }^{15}$ At De Montfort University, this was perceived as a forced opportunity for educators to take a different approach.

De Montfort University's postgraduate pharmacy team are providers of distance learning pharmacy education. The team comprises of Senior Lecturers employed by the institution on a full or part-time basis. The majority of these educators have a portfolio career, some of whom continue to work as pharmacists in patient-facing roles. Their roles are to provide academic leadership and teaching on postgraduate Clinical Pharmacy programmes. Their student profile consists of adult learners ranging from those in their early twenties having just qualified as registered pharmacists to those in their mid-fifties, who have been qualified for decades. The students come from diverse background in terms of ethnicity, culture and experience encompassing clinical, management and leadership roles across primary and secondary care settings. The postgraduate pharmacy team traverse the complexities of students' unique personal and professional circumstances by embracing a student-centred approach. Given the limited requirement for on campus presence and the provisions for distance learning already in-built in the delivery of postgraduate pharmacy education, a 'business as usual' approach could have easily been adopted. The existing provisions to support students included weekly 'helpline' times for students to reach out and contact educators to highlight any personal issues. This arrangement required students to take responsibility and initiate contact with educators. However, continuing this arrangement would have failed to adequately address the significant workplace pressures postgraduate pharmacist students were facing on the frontline working during the pandemic. There were an increasing number of pharmacists reaching out to educators relaying personal stories of the pandemic, their personal loss and grief, the burden and tensions this brought upon them. For many students their acted as a motivational force and was often the one constant in an uncertain climate. These interactions and unique insights forced educators to reflect and reconsider their role and the team felt a moral obligation to assume an enhanced educator role.

This extended role manifested in several ways through a series of initiatives launched by the team, extending their existing student-centred approach to a holistic approach. Acknowledging that this student population is unlikely to access the support structures put in place by the wider university, there were two main strategies. Firstly, students were provided with greater access to the module team, primarily for the purposes of wellbeing check ins and then for enhanced academic support. The second was to motivate and encourage students both professionally and academically via the online learning platform. In the immediate aftermath of the first lockdown, students were given the opportunity to book a one-to-one discussion with the module leader at varying time slots during the week, even outside of conventional office hours and at weekends. Given the large uptake of these one-to-one slots, this micro-level of support was unsustainable for the long term. Consequently, virtual support group sessions facilitated by educators were offered. These were designed to provide and encourage greater peer-to-peer networking and support. The success of these groups resulted in the development of an online community where students could informally interact with one another and be a source of support during the pandemic. Further strategies introduced included more sharing self-help strategies and encouraging learners to reflect on their own mental health and wellbeing. This aligned with wider initiatives such as 'National Stress Awareness Day', where educators reached out and shared messages in an effort to start a dialogue about mental health. Educators made a conscious effort to share aspects of their personal lives that brought them joy in an effort to connect with students. ${ }^{16}$ Informal feedback by students suggested the extended support and strategies deployed were positively received.

As we emerge out of the pandemic, we reflect on whether the support prior to the pandemic was sufficient to cater for working pharmacist students' needs. Embedding mental wellbeing into the curriculum is encouraged for all courses (whether undergraduate or postgraduate) and a number of mental health and wellbeing support mechanisms are available for all students as part of the wider support structures within the University. These mechanisms are 
evolving as a result of the pandemic and increased exposure has provided educators with a greater awareness of how these could be utilised for their respective students. However, it was apparent at the time that those who were qualified health professionals did not feel these were accessible or relevant to them. In line with Muller's ${ }^{6}$ suggestion that healthcare professionals are less likely to access services, educators found that simple signposting to student mental health services would not be sufficient for those students who did not immediately identify themselves as struggling but none the less, required an outlet to discuss the stress they were under. The educators are pharmacists themselves and the support offered was possibly driven because it aligned with their professional identity. They naturally assumed a person-centred wellbeing role where the educator was seen as a 'listening friend' to empathise with students in an open and honest way. Listening to pharmacist's experiences of the pandemic was a crucial component of the interaction and through this, they provided the pharmacist with a source of empathy and comfort. The creation of the online community and peer-to-peer support was a further opportunity and outlet for professionals to share experiences to help manage their mental health and wellbeing during difficult circumstances. The uptake and reception of these interventions highlighted the need, in this instance, for educators to play a greater role in supporting learner's mental health. On reflection, this extended role raised questions about how this model could be formalised or adapted for use in the long-term. Whilst the educators are also healthcare professionals, it is yet to be determined whether the educators were best placed or qualified to provide such support. Under the circumstances, educators felt that these actions were the right thing to do and in the best interest of their students. Going forward, research is needed to investigate the extent to which educators should engage in this work or have the capacity, skills or resource to assume such roles.

\section{CONCLUSION}

Distance learning may be associated with pedagogical theories focussed on delivery of content, demonstration of learning outcomes and assessment. The pandemic exposed a forced opportunity for educators to transform and take on an extended wellbeing role. Through the additional support, a more meaningful learning environment was created, acknowledging the mental health and wellbeing of the learner. If this extended role is to be more widely implemented, further consideration needs to be given to reconfiguring postgraduate distance learning education. Specifically, any extension to existing roles should be developed on the proviso that staff are given both the resources and training to be able to provide this level of support. This case example may also suggest that distance learning theories should incorporate and give more prominence to establishing and responding to the personal circumstances of students and how this may be affecting their performance. It was also a stark reminder that postgraduate students who are also healthcare professionals, practiced in highly pressurised clinical environments prior to the pandemic and, that this cannot to disassociated from their postgraduate learning and development. The pandemic exposed that learners were battling unparalleled stress and trauma impacting on all aspects of their lives for which educators morally recognised and assumed a greater humanistic role. It was recognised that there are clear benefits to developing alternative models of support, learning skills development and that different tactics should be explored to acknowledge this trauma. ${ }^{17}$ More research is needed to theorise the acceptability and feasibility of this new role on a wider scale as the new 'norm' or ensuring educators are not reactive but have a clear vision and plan for future situations where this level of support may be called for.

\section{REFERENCES}

1. Quezada RL, Talbot C, Quezada-Parker KB. From bricks and mortar to remote teaching: A teacher education program's response to COVID-19. Journal of Education for Teaching. 2020;46(4):472-483.

2. Sahi PK, Mishra D, Singh T. Medical education amid the COVID-19 pandemic. Indian Pediatr. 2020;57(7):652-657.

3. Stambough JB, Curtin BM, Gililland JM, et al. The past, present, and future of orthopedic education: lessons learned from the COVID-19 pandemic. J Arthroplasty. 2020;35(7):S60-S64.

4. Kawaguchi-Suzuki M, Nagai N, Akonoghrere RO, Desborough JA. COVID-19 pandemic challenges and lessons learned by pharmacy educators around the globe. Am J Pharm Educ. 2020;84(8).

5. Sahebi A, Nejati B, Moayedi S, Yousefi K, Torres M, Golitaleb M. The prevalence of anxiety and depression among healthcare workers during the COVID-19 pandemic: An umbrella review of meta-analyses. Prog Neuro-Psychopharmacol Biol Psychiatry. 2021:110247.

6. Muller RAE, Stensland RS $\varnothing$, van de Velde, Researcher Stijn. The mental health impact of the covid-19 pandemic on healthcare workers, and interventions to help them: A rapid systematic review. Psychiatry Res. 2020:113441.

7. McCallum BA, Dunkley K, Hotham E, Suppiah V. Bushfires, COVID-19 and Australian community pharmacists: ongoing impact on mental health and wellbeing. International Journal of Pharmacy Practice. 2021;29(2):186-188. 
8. Langran C, Willis S, Hughes L, Mantzourani E, Hall K. Intra and Inter-professional working: how have pharmacists' working practices changed during the COVID-19 pandemic? International Journal of Pharmacy Practice. 2021;29(Supplement_1):i35-i36.

9. Potter JM, Cadiz ML. Addressing Burnout in Pharmacy Residency Programs. Am J Pharm Educ. 2021;85(3).

10. Busch IM, Moretti F, Mazzi M, Wu AW, Rimondini M. What we have learned from two decades of epidemics and pandemics: a systematic review and meta-analysis of the psychological burden of frontline healthcare workers. Psychother Psychosom. 2021:1-13.

11. Sorensen TD, Lin A, Allen DD. Reinventing how pharmacy educators connect as a community. Am J Pharm Educ. 2020;84(6).

12. Malcom DR. Loneliness as a Downstream Concern in a Pandemic (and Post-Pandemic) World. Am J Pharm Educ. 2021;85(4).

13. Shangraw AM, Silvers J, Warholak T, Vadiei N. Prevalence of Anxiety and Depressive Symptoms Among Pharmacy Students. Am J Pharm Educ. 2021;85(2).

14. Atkinson SP. Rethinking personal tutoring systems: the need to build on a foundation of epistemological beliefs. London: BPP University. 2014.

15. Schlesselman LS, Cain J, DiVall M. Improving and Restoring the Well-being and Resilience of Pharmacy Students during a Pandemic. Am J Pharm Educ. 2020;84(6).

16. Brazeau GA, Frenzel JE, Prescott WA. Facilitating wellbeing in a turbulent time. Am J Pharm Educ. 2020;84(6).

17. Persky AM, Fuller KA, Jarstfer M, Rao K, Rodgers JE, Smith M. Maintaining core values in postgraduate programs during the COVID-19 pandemic. Am J Pharm Educ. 2020;84(6). 